\title{
THE LOCAL THEORY OF ELASTOPLASTIC DEFORMATION PROCESSES AND THE STABILITY BEYOND ELASTIC LIMITS OF THIN-WALLED STRUCTURES SUBJECTED TO COMPLEX LOADING
}

\author{
DAO HÜY BICH \\ Hanoi State University
}

\begin{abstract}
SUMMARY. The paper is concerned with the complete constitutive relations of elastoplastic deformation process theory. Using this theory the stability beyond elastic limits of thin-walled structures subjected to complex loading is analysed. The proposed method of loading parameter is a combination of numerical and analytical solutions. Calculations have been carried out for rectangular plates and cylindrical shells in order to compare this method and its results with other theoretical and experimental works.
\end{abstract}

\section{CONSTITUTIVE RELATIONS OF THE LOCAL THEORY OF ELASTOPLASTIC DEFORMATION PROCESSES}

The analysis of stress-strain states or the stability of components or structures subjected to various complex loading beyond limits of elasticity requires a plasticity theory which can describe complex elastoplastic processes of deformation. The theory of elastoplastic deformation processes, based on Ilyushin's postulate of isotropy satisfes this requirement. But up to now the stressutrain relationship has contained undetermined functionals.

Assuming some hypothesis, we have constructed a complete stressustrain relationship of the local theory of elastoplastic deformation processes using hypothesis of local determinancy and complanarity $[1,2]$ :

$$
\begin{aligned}
\dot{S}_{i j} & =-\frac{2}{3} \frac{\sigma_{u} f}{\sin \theta} \dot{e}_{i j}+\left(\frac{\psi}{\cos \theta}+\frac{\sigma_{u} f}{\sin \theta}\right) \frac{S_{m n} \dot{e}_{m n}}{\sigma_{u}^{2}} S_{i j} \\
\dot{\sigma} & =3 K \dot{e} .
\end{aligned}
$$

This theory containes two material functions $f$ and $\psi$ depending upon the materials used. They are determined from experimental data [2]

$$
\begin{aligned}
& f \equiv f\left(\sigma_{t}, \theta, s\right)=-\frac{1}{s} \sin \theta\left[1+\left(\frac{3 G s}{\sigma_{u}}-1\right)\left(\frac{1-\cos \theta}{2}\right)^{\prime \prime}\right] \\
& \psi \equiv \psi(\theta, s)=\phi^{\prime}(s) \cos \theta-\left(3 G-\phi^{\prime}\right)\left(\frac{1-\cos \theta}{2}\right)^{\beta}, \quad \alpha \geq 1, \quad \beta>1,
\end{aligned}
$$

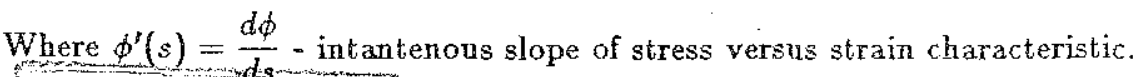

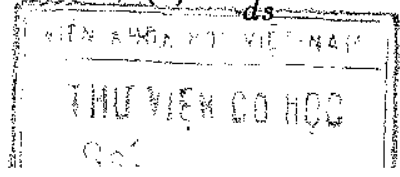


It is significant that $f$ and $\psi$ can be applied for all active and passive deformation processes, i.e. the stress-strain relationship (1.1), (1.2) can describe all deformation processes with complex loading (not only loading, but unloading as well). The relationship for simple loading process, process with small and average curvature, unloading process and Prandtl-Reuss relations are considered as particular cases of this theory.

\section{a. Simple loading process}

For this process $\theta=0$,

$$
\lim _{\theta \rightarrow 0} \frac{f}{\sin \theta}=-\frac{1}{s}, \quad \lim _{\theta \rightarrow 0} \psi=\phi^{\prime}(s),
$$

the relations (1.1)-(1.2) become

$$
\dot{S}_{i j}=\frac{2}{3} \frac{\sigma_{u}}{s} \dot{e}_{i j}+\left(\phi^{\prime}-\frac{\sigma_{u}}{s}\right) \frac{S_{i j}}{\sigma_{u}} v_{u}
$$

Otherwise, according to the small elastoplastic deformation theory for simple loading

$$
S_{i j}=\frac{2 \sigma_{u}}{3 \varepsilon_{t z}} \varepsilon_{i j}
$$

we obtain

$$
\dot{S}_{i j}=\frac{2 \sigma_{u}}{3 \varepsilon_{u}} \dot{e}_{i j}+\frac{2}{3}\left(\frac{d \sigma_{u}}{d \varepsilon_{u}}-\frac{\sigma_{u}}{\varepsilon_{u}}\right) \frac{S_{i j}}{\sigma_{u}} \dot{\varepsilon}_{u}
$$

Since in this case

$$
s=\varepsilon_{u}, \quad \dot{s}=\dot{\varepsilon}_{u}=v_{u}, \quad \frac{d \sigma_{u}}{d \varepsilon_{u}}=\frac{d \sigma_{u}}{d s}=\phi^{\prime}(s),
$$

hence the relation (1.4) reduces to (1.3)

In the elastic stage $\frac{\sigma_{u}}{s}=3 G, \phi^{\prime}=3 G$, from (1.3) we obtain Hookean relationship

$$
\dot{S}_{i j}=2 G \dot{e}_{i j}
$$

\section{b. Unloading process}

The unloading process occurs when $\theta=\pi$, i.e. the direction of the tangent to the continuing deformation trajectory is opposite to the stress vector at considered point. Since

$$
\lim _{\theta \rightarrow+\pi} \frac{f}{\sin \theta}=-\frac{3 G}{\sigma_{u}}, \quad \lim _{\theta \rightarrow \pi} \psi=-3 G
$$

hence the relatios (1.1)-(1.2) become

$$
\dot{S}_{i j}=2 G \dot{e}_{i j}
$$

\section{c. Deformation process with average curvature}

In this process the value of angle $\theta$ is small. Restricting to the second-order small values we obtain from (1.2)

$$
f=-\frac{1}{s} \sin \theta \approx-\frac{\theta}{s}, \quad \psi=\phi^{\prime} \cos \theta \approx \phi^{\prime}\left(1-\frac{\theta^{2}}{2}\right)
$$


Substituting into the relations (1.1)-(1.2) gives

$$
\dot{S}_{i j}=\frac{2}{3} \frac{\sigma_{u}}{s} \dot{e}_{i j}+\left(\phi^{\prime}-\frac{\sigma_{u}}{s}\right) \frac{S_{m n} \dot{e}_{m n+}}{\sigma_{u}^{2}} S_{i j} .
$$

The relation (1.7) is a generalization of Prandtl-Reuss relation for perfectly plastic material and Prager relation for plastic strain-hardening material.

\section{THE STABILITY OF THIN-WALLED STRUCTURES SUBJECTED TO COMPLEX LOADING}

In recent years large development in the elastoplastic analysis of thin-walled structures has been observed. But there is no an estimation about the influence of complex loading on the stability of structures.

Suppose that components of structures are subjected to external forces which are considered as loads depending on some parameter. $t$. When $t$ varies, the deformation process occured by these loads in structures may be simple or complex.

The instability of the structures is expressed that with $t=t_{k}$ external load reaches some special value, by this load a stress state $\sigma_{i j}^{k}$ and a strain state $\varepsilon_{i j}^{k}$ occur respectively in the structure such that before and up to this state the deformation process is still determined one-by-one, but after that there exist neighbouring states, i.e. there exists a bifurcation of equilibrium states.

One of the main aims of the stability problem is to determine this value $t_{k}$. The value $t_{k}$ is called a critical value of loading parameter, and respectively external load is called a critical load. As shown later, the critical load depents on the complexity of loading process.

\section{a. Pre-buckling process}

Suppose that a thin-walled structure is subjected to complex loading. At any moment $t$ there exists a membrane plane stress state in the structure

$$
\sigma_{11}, \quad \sigma_{32}, \quad \sigma_{23} \neq 0, \quad \sigma_{33}=\sigma_{31}=\sigma_{32}=0
$$

so that

$$
\sigma=\frac{1}{3}\left(\sigma_{11}+\sigma_{33}\right), \quad \sigma_{24}=\left(\sigma_{11}^{2}+\sigma_{33}^{2}-\sigma_{11} \sigma_{33}+3 \sigma_{13}^{2}\right)^{1 / 2}
$$

The strain tensor is determined from (1.1)-(1.2) (with $\nu=1 / 2$ ) in combination with bonndary conditions:

$$
\dot{\sigma}_{i j}=-\frac{2}{3} \frac{\sigma_{u} f}{\sin \theta}\left(\dot{\varepsilon}_{i j}+\delta_{i j} \dot{\varepsilon}_{k k}\right)+\left(\frac{\psi}{\cos \theta}+\frac{\sigma_{i j} f}{\sin \theta}\right) \frac{\sigma_{k t} \dot{\varepsilon}_{k \ell}}{\sigma_{i k}^{2}} \sigma_{i j}
$$

where

$$
\cos \theta=\frac{\sigma_{i j} \dot{\varepsilon}_{i j}}{\sigma_{u} v_{u}}, \quad v_{u} \equiv \frac{d s}{d t}=\frac{2}{\sqrt{3}}\left(\dot{\varepsilon}_{11}^{2}+\dot{\varepsilon}_{32}^{2}+\dot{\varepsilon}_{k 1} \dot{\varepsilon}_{32}+\dot{\varepsilon}_{12}^{2}\right)^{1 / 2} .
$$

\section{b. Equations of stability}

Suppose, that at the moment $t_{k}$ a bifurcation of equilibrinm states appears such that with an infinitesimal small increment of external load there are possible increments of deformation (including the bending deformation) in the structure 


$$
\delta \bar{\epsilon}_{i j}=\delta \varepsilon_{i j}^{*}-Z \delta \chi_{i j}
$$

where

$$
\delta \varepsilon_{i j}^{*}=\frac{1}{2}\left(\frac{\partial \delta u_{i}}{\partial x_{j}}+\frac{\partial \delta u_{j}}{\partial x_{i}}\right)-k_{i j} \delta w, \quad \delta \chi_{i j}=\frac{\partial^{2} \delta w}{\partial x_{i} \partial x_{j}},
$$

$\delta u_{i}, \delta w$ - increment of in-plane displacement and deflection of middle surface.

$\delta \chi_{i j}$ - increment of curvature and torsion associated with the instability.

$k_{i j}$ - principal curvature of the shell, $\left(k_{13}=0\right)$.

Respectively, we determine stress increments according to (1.1), (1.2) (with $\nu=1 / 2$ ):

$$
\delta \sigma_{i j}=\frac{2}{3} A\left(\delta \varepsilon_{i j}+\delta_{i j} \delta \varepsilon_{k k}\right)+(P-A) \frac{\sigma_{k \ell} \delta \varepsilon_{k \ell}}{\sigma_{u}^{2}} \sigma_{i j} .
$$

where

$$
\begin{aligned}
& A=-\frac{\sigma_{u} f}{\sin \theta}=\frac{\sigma_{u}}{s}+\left(3 G-\frac{\sigma_{u}}{s}\right)\left(\frac{1-\cos \theta}{2}\right)^{\alpha}, \\
& P=\frac{\psi}{\cos \theta}=\phi^{\prime}(s)-\frac{1}{\cos \theta}\left(3 G-\phi^{\prime}\right)\left(\frac{1-\cos \theta}{2}\right)^{\beta} .
\end{aligned}
$$

In the case of the deformation process with average curvature $A$ and $B$ become

$$
A \equiv N=\frac{\sigma_{u}}{s}, \quad P \equiv \phi^{\prime} .
$$

Using (2.3), (2.4) we write increments of membrane forces and bending moments in the form

$$
\begin{aligned}
\delta T_{i j}=\int_{-h / 2}^{h / 2} \delta \sigma_{i j} d Z= & \frac{2}{3} A_{1}\left(\delta \varepsilon_{i j}^{*}+\delta_{i j} \delta \varepsilon_{k k}^{*}\right)-\frac{2}{3} A_{2}\left(\delta \chi_{i j}+\delta_{i j} \delta \chi_{k k}\right)+ \\
& +\frac{\sigma_{i j}}{\sigma_{u}}\left[\left(P_{1}-A_{1}\right) \varepsilon-\left(P_{2}-A_{2}\right) \chi\right] \\
\delta M_{i j}=\int_{-h / 2}^{h / 2} \delta \sigma_{i j} Z d Z= & \frac{2}{3} A_{2}\left(\delta \varepsilon_{i j}^{*}+\delta_{i j} \varepsilon_{k k}^{*}\right)-\frac{2}{3} A_{3}\left(\delta \chi_{i j}+\delta_{i j} \delta \chi_{k k}\right)+ \\
& +\frac{\sigma_{i j}}{\sigma_{u}}\left[\left(P_{2}-A_{2}\right) \varepsilon-\left(P_{3}-A_{3}\right) \chi\right]
\end{aligned}
$$

where

$$
P_{m}=\int_{-h / 2}^{h / 2} P Z^{m-1} d Z, \quad A_{m}=\int_{-h / 2}^{h / 2} A Z^{m-1} d Z, \quad \varepsilon=\frac{\sigma_{i j}}{\sigma_{u}} \delta \varepsilon_{i j}^{*}, \quad \chi=\frac{\sigma_{i j}}{\sigma_{u}} \delta \chi_{i j} .
$$

Written quantities satisfy the stability equations of plates or shells

$$
\begin{aligned}
& \frac{\partial \delta T_{i j}}{\partial x_{j}}=0, \\
& \frac{\partial^{2} \delta M_{i j}}{\partial x_{i} \partial x_{j}}+T_{i j} \delta \chi_{i j}+k_{i j} \delta T_{i j}=0, \\
& \frac{\partial^{2} \delta \varepsilon_{11}^{*}}{\partial x_{2}^{2}}+\frac{\partial^{2} \delta \varepsilon_{22}^{*}}{\partial x_{1}^{2}}-2 \frac{\partial^{2} \delta \varepsilon_{13}^{*}}{\partial x_{1} \partial x_{2}}=k_{11} \frac{\partial^{2} \delta w}{\partial x_{2}^{2}}+k_{23} \frac{\partial^{2} \delta w}{\partial x_{1}^{2}} .
\end{aligned}
$$


Equation (2.1)-(2.7) form a system of fundamental equations for solving a stability problem of thin plates or shells

\section{CALCULATIONS FOR RECTANGULAR PLATES AND CYLINDRICAL SHELLS}

The stability of rectangular plates subjected to complex biaxial compression was analysed in [3].

Now let's consider the stability problem of a cylindrical shell of radius $R$, thickness $h$ and length $L$ subjected to complex loading. In this case we choose $x$ lying along the generatrix of the shell, $y=R \theta$ and $Z$ - in the direction of the normal, so that $k_{11}=0, k_{32}=1 / R$.

Suppose that the deformation process in the shell is determined by the constitutive relations (2.2), (2.5). Since $A$ and $P$ do not depend on variable $Z$, so that $A_{2}=P_{2}=0, \delta T_{i j}$ depend only on $\delta \varepsilon_{i j}^{*}$, and $\delta M_{i j}$ - only on $\delta \chi_{i j}$.

Stability equations (2.7) become

$$
\begin{aligned}
\alpha_{1} \frac{\partial^{4} \delta w}{\partial x^{4}} & +\alpha_{2} \frac{\partial^{4} \delta w}{\partial x^{3} \partial y}+\alpha_{3} \frac{\partial^{4} \delta w}{\partial x^{2} \partial y^{2}}+\alpha_{4} \frac{\partial^{4} \delta w}{\partial x \partial y^{3}}+ \\
& +\alpha_{5} \frac{\partial^{4} \delta w}{\partial y^{4}}-\frac{9}{N h^{2}}\left(\sigma_{11} \frac{\partial^{2} \delta w}{\partial x^{2}}+\sigma_{32} \frac{\partial^{2} \delta w}{\partial y^{2}}+2 \sigma_{13} \frac{\partial^{2} \delta w}{\partial x \partial y}-\frac{1}{R} \frac{\partial^{2} \varphi}{\partial x^{2}}\right)=0 \\
\beta_{1} \frac{\partial^{4} \varphi}{\partial x^{4}} & +\beta_{3} \frac{\partial^{4} \varphi}{\partial x^{3} \partial y}+\beta_{3} \frac{\partial^{4} \varphi}{\partial x^{2} \partial y^{2}}+\beta_{4} \frac{\partial^{4} \varphi}{\partial x \partial y^{3}}+\beta_{5} \frac{\partial^{4} \varphi}{\partial y^{4}}+\frac{N}{R} \frac{\partial^{2} \delta w}{\partial x^{2}}=0
\end{aligned}
$$

where

$$
\begin{aligned}
& \delta T_{11}=\frac{\partial^{2} \varphi}{\partial y^{2}}, \quad \delta T_{22}=\frac{\partial^{2} \varphi}{\partial x^{2}}, \quad \delta T_{12}=-\frac{\partial^{2} \varphi}{\partial x \partial y} \\
& \alpha_{1}=1-\frac{3}{4}\left(1-\frac{\phi^{\prime}}{N}\right) \frac{\sigma_{11}^{2}}{\sigma_{u}^{2}}, \quad \alpha_{2}=-3\left(1-\frac{\phi^{\prime}}{N}\right) \frac{\sigma_{11} \sigma_{12}}{\sigma_{u}^{2}} \\
& \alpha_{3}=2-\frac{3}{4}\left(1-\frac{\phi^{\prime}}{N}\right) \frac{\sigma_{13}^{2}}{\sigma_{u}^{2}}-\frac{3}{2}\left(1-\frac{\phi^{\prime}}{N}\right) \frac{\sigma_{x 1} \sigma_{22}}{\sigma_{u t}^{2}} \text {, } \\
& \alpha_{4}=-3\left(1-\frac{\phi^{t}}{N}\right) \frac{\sigma_{12} \sigma_{32}}{\sigma_{u}^{2}}, \quad \alpha_{5}=1-\frac{3}{4}\left(1-\frac{\phi^{t}}{N}\right) \frac{\sigma_{23}^{2}}{\sigma_{u}^{2}} \\
& \beta_{3}=1+\frac{1}{4}\left(\frac{N}{\phi^{\prime}}-1\right)\left(\frac{2 \sigma_{33}-\sigma_{21}}{\sigma_{u}}\right)^{2}, \quad \beta_{3}=-3\left(\frac{N}{\phi^{\prime}}-1\right) \frac{\sigma_{23}\left(2 \sigma_{33}-\sigma_{23}\right)}{\sigma_{u}^{2}}, \\
& \beta_{3}=2+\frac{1}{2}\left(\frac{N}{\phi^{\prime}}-1\right) \frac{\left(2 \sigma_{11}-\sigma_{22}\right)\left(2 \sigma_{23}-\sigma_{13}\right)}{\sigma_{u}^{2}}+9\left(\frac{N}{\phi^{\prime}}-1\right) \frac{\sigma_{12}^{2}}{\sigma_{u t}^{2}}, \\
& \beta_{4}=-3\left(\frac{N}{\phi^{\prime}}-1\right) \frac{\sigma_{12}\left(2 \sigma_{11}-\sigma_{22}\right)}{\sigma_{u}^{2}}, \quad \beta_{s}=1+\frac{1}{4}\left(\frac{N}{\phi^{\prime}}-1\right)\left(\frac{2 \sigma_{11}-\sigma_{32}}{\sigma_{u}^{2}}\right)^{2} \text {. }
\end{aligned}
$$

Satisfying kinematic boundary conditions with butt-ends simply supported, we can find solutions of the form

$$
\delta w=A \sin \left(\frac{m \pi x}{L}-\frac{n y}{R}\right), \quad \varphi=B \sin \left(\frac{m \pi x}{L}-\frac{n y}{R}\right)
$$


Consider some particular cases:

a. Cylindrical shell subjected to compression along generatrix

In this case

$$
\begin{aligned}
& \sigma_{11}=-p, \quad \sigma_{32}=\sigma_{12}=0, \quad \sigma_{u}=\left|\sigma_{11}\right| \\
& \alpha_{1}=\frac{1}{4}+\frac{3}{4} \frac{\phi^{\prime}}{N}, \quad \alpha_{3}=2, \quad \alpha_{3}=1, \quad \alpha_{3}=\alpha_{4}=0, \\
& \beta_{1}=\frac{3}{4}+\frac{1}{4} \frac{N}{4 \phi^{\prime}}, \quad \beta_{3}=3-\frac{N}{\phi^{\prime}}, \quad \beta_{5}=\frac{N}{\phi^{\prime}}, \quad \beta_{3}=\beta_{4}=0 .
\end{aligned}
$$

Substituting these values and $\delta w, \varphi$ by (3.3) into stability equations (3.1), (3.2) and taking in account of the existence of nontrivial solution, we receive a relation for finding critical force

or

$$
i^{2}=\frac{N}{p \lambda_{m}^{2}}\left(\alpha_{1} \lambda_{m}^{4}+\alpha_{3} \lambda_{m}^{2} n^{2}+\alpha_{5} n^{4}+\frac{\lambda_{m}^{4} z^{2}}{\beta_{1} \lambda_{m}^{4}+\beta_{3} \lambda_{m}^{2} n^{2}+\beta_{5} n^{4}}\right)
$$

$$
\dot{8}^{2}=\frac{N}{p} \frac{\left(\alpha_{1} \lambda_{m}^{4}+\alpha_{3} \lambda_{m}^{2} n^{2}+\alpha_{5} n^{4}\right)\left(\beta_{1} \lambda_{m}^{4}+\beta_{3} \lambda_{m} 2 n^{2}+\beta_{5} n^{4}\right)}{\lambda_{m}^{2}\left(\beta_{1} \lambda_{m}^{4}+\beta_{3} \lambda_{m}^{2} n^{2}+\beta_{5} n^{4}\right)-\lambda_{m}^{4} \frac{N}{p}}
$$

where

$\lambda_{m t}=m \pi R / L$

$i=3 R / h-$ stiffness of the shell,

Denoting by $X=n^{2}, Y=\lambda_{m}^{2} / n^{2}$ rewrite (3.4) as follows

$$
i^{2}=\frac{N}{p} \frac{X^{2}\left(\alpha_{1} Y+\alpha_{3}+\frac{\alpha_{5}}{Y}\right)\left(\beta_{1} Y+\beta_{3}+\frac{\beta_{5}}{Y}\right)}{X\left(\beta_{1} Y+\beta_{3}+\frac{\beta_{5}}{Y}\right)-\frac{N}{p}}
$$

Mininizing (3.5)

$$
\frac{\partial i^{2}}{\partial X}=0, \quad \frac{\partial i^{2}}{\partial Y}=0
$$

gives us

$$
i^{2}=4 \frac{N^{2}}{p^{2}} \frac{\phi^{\prime}}{N} \frac{2+\sqrt{1+3 \frac{\phi^{\prime}}{N}}}{3 \frac{\phi^{\prime}}{N}-1+\sqrt{1+3 \frac{\phi^{\prime}}{N}}}
$$

Hence

where $N=\sigma_{u} / s$

$$
i=2 \frac{N}{p} \sqrt{\frac{\phi^{\prime}}{N}} \sqrt{\frac{2+\sqrt{1+3 \frac{\phi^{\prime}}{N}}}{3 \frac{\phi^{\prime}}{N}-1+\sqrt{1+3 \frac{\phi^{\prime}}{N}}}}
$$

Since $s$ is determined from simple expression 


$$
\sigma_{u}=p=\phi(s), \quad \text { so that } s=\phi^{-1}(p)
$$

then critical force $p_{k}$ can be found from (3.6).

For a long cylindrical shell $\lambda_{m}^{2} \ll n^{2}$ we obtain

$$
i^{2}=\frac{N}{p \lambda_{m}^{2}}\left(\alpha_{s} n^{4}+\frac{\lambda_{m}^{4} i^{2}}{\beta_{s} n^{4}}\right)
$$

A minimization gives

$$
i=\frac{2}{p} \sqrt{N \phi^{\prime}}
$$

b. Cylindrical shell subjected to compression and external pressure

Pre-buckling process is of the form

$$
\sigma_{11}=-p(t), \quad \sigma_{33}=-\tilde{q} \frac{R}{h}=-q(t), \quad \sigma_{13}=0, \quad \sigma_{u}^{2}=p^{2}-p q+q^{2},
$$

where $t$ is a loading parameter. Respectively, deformation increments are determined by following equations

$$
\begin{aligned}
& \dot{\varepsilon}_{11}=\frac{1}{N}\left(-\dot{p}+\frac{1}{2} \dot{q}\right)-\left(\frac{1}{\phi^{\prime}}-\frac{1}{N}\right) \frac{\left(p \dot{p}+q \dot{q}-\frac{1}{2} p \dot{q}-\frac{1}{2} q \dot{p}\right)\left(p-\frac{1}{2} q\right)}{p^{2}-p q+q^{2}} \\
& \dot{\varepsilon}_{32}=-\frac{1}{N}\left(\dot{q}-\frac{1}{2} \dot{p}\right)-\left(\frac{1}{\phi^{\prime}}-\frac{1}{N}\right) \frac{\left(p \dot{p}+q \dot{q}-\frac{1}{2} p \dot{q}-\frac{1}{2} q \dot{p}\right)\left(q-\frac{1}{2} p\right)}{p^{2}-p q+q^{2}}
\end{aligned}
$$

where

$$
N=\frac{\sigma_{u}}{s}=\frac{1}{s}\left(p^{2}-p q+q^{2}\right)^{1 / 2} .
$$

The arc-length of the deformation trajectory is found by

$$
\frac{d s}{d t}=\frac{2}{\sqrt{3}}\left(\dot{\varepsilon}_{11}^{2}+\dot{\varepsilon}_{23}^{2}+\dot{\varepsilon}_{11} \dot{\varepsilon}_{23}\right)^{1 / 2} \equiv F(s, p, q)
$$

Since $p, q$ depend on loading parameter $p=p(t), q=q(t)$, so from (3.8) $s$ is determined as a function of $t$.

In this cases the coefficients of the stability equations (3.1), (3.2) are the following

$$
\begin{aligned}
& \alpha_{2}=1-\frac{3}{4}\left(1-\frac{\phi^{3}}{N}\right) \frac{p^{2}}{p^{2}-p q+q^{2}}, \\
& \alpha_{3}=2-\frac{3}{2}\left(1-\frac{\phi^{\prime}}{N}\right) \frac{p q}{p^{2}-p q+q^{2}}, \\
& \alpha_{5}=1-\frac{3}{4}\left(1-\frac{\phi^{\prime}}{N}\right) \frac{p^{2}}{p^{2}-p q+q^{2}}, \\
& \alpha_{3}=\alpha_{4}=0 .
\end{aligned}
$$




$$
\begin{aligned}
& \beta_{1}=1+\frac{1}{4}\left(\frac{N}{\phi^{\prime}}-1\right) \frac{(2 q-p)^{2}}{p^{2}-p q+q^{2}} \\
& \beta_{3}=2+\frac{1}{2}\left(\frac{N}{\phi^{\prime}}-1\right) \frac{(2 p-q)(2 q-p)}{p^{2}-p q+q^{2}} \\
& \beta_{5}=1+\frac{1}{4}\left(\frac{N}{\phi^{\prime}}-1\right) \frac{(2 p-q)^{2}}{p^{2}-p q+q^{2}} \\
& \beta_{3}=\beta_{4}=0
\end{aligned}
$$

By the same method we get an expression for finding critical load

$$
i^{2}=\frac{N}{f_{m}^{2}+q n^{2}}\left(\alpha_{1} \lambda_{m}^{4}+\alpha_{3} \lambda_{m}^{2} n^{2}+\alpha_{5} n^{4}+\frac{\lambda_{m}^{4} i^{2}}{\beta_{1} \lambda_{m}^{4}+\beta_{3} \lambda_{m}^{2} n^{2}+\beta_{5} n^{4}}\right) .
$$

Using previous notation $X, Y$ we rewrite

$$
3^{2}=\frac{N\left(\alpha_{1} Y+\alpha_{3}+\frac{\alpha_{s}}{Y}\right)\left(\beta_{1} Y+\beta_{3}+\frac{\beta_{5}}{Y}\right) X^{2}}{X\left(p+\frac{q}{Y}\right)\left(\beta_{1} Y+\beta_{3}+\frac{\beta_{5}}{Y}\right)-N} .
$$

Minimization of $i^{2}$ gives

$$
\begin{aligned}
G(s, p, q, Y) \equiv & \left(\beta_{1} Y+\beta_{3}+\frac{\beta_{5}}{Y}\right)\left(\alpha_{1}-\frac{\alpha_{5}}{Y^{2}}\right)-\left(\alpha_{1} Y+\alpha_{3}+\frac{\alpha_{5}}{Y}\right)\left(\beta_{2}-\frac{\beta_{5}}{Y^{2}}\right)+ \\
& +2\left(\alpha_{1} Y+\alpha_{3}+\frac{\alpha_{5}}{Y}\right)\left(\beta_{1} Y+\beta_{3}+\frac{\beta_{5}}{Y}\right) \frac{q}{Y^{2}\left(p+\frac{q}{Y}\right)}=0 \\
i & =\frac{2 N}{p+\frac{q}{Y}}\left(\frac{\alpha_{1} Y+\alpha_{3}+\frac{\alpha_{5}}{Y}}{\beta_{1} Y+\beta_{3}+\frac{\beta_{5}}{Y}}\right)^{1 / 2} \equiv H(s, p, q, Y) .
\end{aligned}
$$

From equation (3.10) $Y$ is determined as a function of $s, p, q$ and then substituted into (3.11). Expressing $p, q$ through loading parameter $t$, from (3.8) and (3.11) we can find critical value of loading parameter $t_{k}$ by numerical calculation.

Critical loads are the following

$$
p_{k}=p\left(t_{k}\right), \quad q_{k}=q\left(t_{k}\right)
$$

For a long cylindrical shell $\lambda_{n n}^{2} \ll n^{2}$ we obtain

$$
i^{2}=\frac{N}{p \lambda_{m}^{2}+q n^{2}}\left(\alpha_{s} n^{4}+\frac{\lambda_{m}^{4} i^{2}}{\hat{f}_{5} n^{4}}\right)
$$

Minimization of $i^{2}$ gives us

$$
i=\frac{2 N}{p} \sqrt{\frac{1-\frac{3}{4}\left(1-\frac{\phi^{\prime}}{N}\right) \frac{q^{2}}{p^{2}-p q+q^{2}}}{1+\frac{1}{4}\left(\frac{N}{\phi^{\prime}}-1\right) \frac{(2 p-q)^{2}}{p^{2}-p q+q^{2}}+4 N \frac{q}{p^{2}}}} \equiv H(s, p, q) .
$$

Expressing $p=p(t), q=q(t)$, we can find the critical value of loading parameter $t_{k}$ from (3.12) and (3.8) by numerical calculation. 


\section{CONCLUSIONS}

1. Constitutive relations are formulated completely. Contained in these relations material functions are already determined.

2. The local theory of elastoplastic deformation processes can be applied to the stability problems of thin-walled structures, when both Pre-buckling process and Post-buckling processes are complicated.

3. Proposed method of loading parameter gives a way to solve stability problem for all types of loading.

4. Complex loading process has an essential influence on the stability of structures. Critical loads are lower than that is for simple loading.

\section{REFERENCES}

1. Dao Huy Bich. Uniqueness theorem of boundary value problem of plasticity using the hypothesis of local determinancy. Mechanics of Solids, V. 17, No 1, 1982, pp. 110-115 (Translated by Allerton Press Inc., Math. Reviews 83h. 73027, 1983).

2. Dao Huy Bich. Research of the local theory of elastoplastic deformation processes. Dr. Sc. Thesis, The State University of Moscow, 1988, (in Russian)

3. Dao Huy Bich. Influence of complex loading on the stability outside elastic limits of thin plates. Journal of Mechanics (Tạp chí Co Học) Vol. 11, No 3, 1989, pp. 1-7 (in vietnamese).

Received December 28, 1991

\section{LÝ THUYÉT QUÁ TRINH BIẾN DANG DÀN DÉO \\ VÀ BÀI TOÁN ỔN ĐỊNH NGOÀI GIÓI HẠN ĐÀN HỒI \\ CƯ A CÁC KÉT CẤU THÀNH MÓNG CHỊU TẢT PHÚC TẠP}

Dựa trên định đề đẳng hướng và giả thuyết xác định địa phương tác già đã xây dựng ảược dạng hoàn chính của lý thuyết quá trình biến dạng dàn dẻo. Lý thuyết chứa đựng hai hàm vật liệu mò tả tính chất vô hướng và tính chất vectơ của vật liệu. Các hàm này cūng đã được thiết lập tù̀ các số liệu thực nghiệm.

Sử dụng lý thuyết trên vào bài toán ổn định ngoài giới hạn đàn hồi của kết cấu thành móng khi quá trình biến dạng trước và sau khi mất ổ định đêu là phức tạp. Đưa ra phương pháp giải loại bài toán này thông qua tham số tải. Giải bài toán cụ thể về bân mỏng chữ nhật và vỏ trụ chịu tải phức tạp. 